\title{
Questes
}

Revue pluridisciplinaire d'études médiévales

Journée d'étude 1 - Trier, classer, organiser | 2022

Trier, classer, organiser

\section{La liste comme structure du savoir dans les traductions françaises du Lilium medicinae de Bernard de Gordon}

\section{Adeline Sanchez}

\section{OpenEdition}

Journals

Édition électronique

URL : https://journals.openedition.org/questes/6003

DOI : $10.4000 /$ questes.6003

ISSN : 2109-9472

Éditeur

Les Amis de Questes

Édition imprimée

Pagination: 156-172

ISSN : 2102-7188

Référence électronique

Adeline Sanchez, « La liste comme structure du savoir dans les traductions françaises du Lilium medicinae de Bernard de Gordon », Questes [En ligne], Journée d'étude 1 - Trier, classer, organiser I 2022, mis en ligne le 31 janvier 2022, consulté le 23 février 2022. URL : http://

journals.openedition.org/questes/6003 ; DOI : https://doi.org/10.4000/questes.6003 


\title{
La liste comme structure du savoir dans les traductions françaises du Lilium medicinae de Bernard de Gordon
}

\author{
Adeline SANCHEZ \\ Sorbonne Université
}

Dans un domaine qui se veut aussi pragmatique et aussi codifié que celui de la médecine, on peut se demander quelle place prend l'effet de liste et quel est son rôle. Entre savoir théorique et pratique, la practica du maître en médecine montpelliérain Bernard de Gordon ${ }^{1}$ est un terrain de recherche intéressant sur ces questions. Le succès de son Lilium medicinae ${ }^{2}$, tant pour sa médecine que pour la forme de la practica en elle-même, s'explique, entre autres choses, par un exposé clair et synthétique, malgré une volonté d'embrasser l'ensemble du savoir médical à des fins didactiques et pratiques. L'énumération est alors un procédé que l'on s'attend à rencontrer afin de parcourir l'ensemble de la matière et des situations ou cas envisagés. Mais cette œuvre, avant d'être un outil de pratique, est un exercice de composition universitaire : héritière du compendium ${ }^{3}$, dont elle est le pendant pratique, elle restitue une médecine scolastique, a été composée par un universitaire et s'inscrit

\footnotetext{
${ }^{1}$ Bernard de Gordon est maître à l'université de Montpellier de 1283 à 1308 . À son sujet, voir Luke Demaitre, Doctor Bernard de Gordon: Professor and Practitioner, Toronto, Pontifical Institute of Mediaeval Studies, 1980.

${ }^{2}$ Composée entre 1305 et 1310 , cette œuvre est traduite dès le XIV ${ }^{\mathrm{e}}$ siècle en langue vernaculaire à travers l'Europe. Actuellement, nous conservons, sous la forme de fragments ou de versions intégrales, quatre-vingts manuscrits du Lilium medicinae en latin et vingt-six traductions, dont nos trois versions françaises.

${ }^{3}$ Le compendium est une composition universitaire visant à faire la synthèse théorique du savoir d'une science.
} 
ainsi dans une tradition rhétorique particulière. L'enjeu pratique de la liste se joue peut-être dans un effort de différenciation de l'effet rhétorique du procédé et de sa valeur dans l'organisation de la matière médicale, deux usages de l'effet de liste. Son absence ou sa présence au sein des différents témoins de notre corpus fait donc sens : les fragments manuscrits sont supposés d'utilité ou d'emploi direct, et les imprimés français sont à considérer comme de véritables manuels conservant l'œuvre intégrale. Nous conservons à l'heure actuelle trois fragments manuscrits du Lys de medecine, au sein de compilations à vocation médicale et/ou chirurgicale ${ }^{4}$. Les traductions imprimées françaises représentent, quant à elles, une tradition unique ${ }^{5}$. S'interroger sur l'énumération et l'effet de liste dans les versions françaises de cette practica latine revient donc à s'intéresser à la structuration du savoir médical et aux caractéristiques et/ou modalités de transmission et réception de ce savoir.

\section{Classer et ordonner la matière}

Enrique Montero Cartelle relève le lien de parenté entre compendium et practica en précisant que cette dernière forme reprend de la première la méthode d'exposition brève, l'organisation de la tête aux pieds et la visée didactique de l'ensemble $^{6}$. Notre practica montpelliéraine ne déroge pas à ces observations, mais, comme le

\footnotetext{
${ }^{4}$ Il s'agit des manuscrits français 1288 (A), 1327 (B) et 19989 (C), datés du $\mathrm{XV}^{\mathrm{e}}$ siècle, conservés à la $\mathrm{BnF}$.

${ }^{5}$ Les imprimés français retenus ici sont ceux conservés à la BnF et à la Bibliothèque interuniversitaire de Montpellier, édités respectivement en 1495 à Lyon par un anonyme (D) et en 1509 par Jehan Petit à Paris (E). L'imprimé latin retenu est conservé à la $\mathrm{BnF}$ et fut imprimé en 1480 à Naples par Francisco del Tuppo. Les colophons mentionnent l'origine de cette tradition, un manuscrit qui est à l'heure actuelle perdu.

${ }^{6}$ Enrique Montero Cartelle, Tipologia de la literatura médica latina. Antigüedad, Edad Media, Renacimiento, Turnhout, Brepols, 2010, p. 57-64.
} 
mentionne Geneviève Dumas, ce genre de la littérature médicale n'est pas systématique et dépend avant tout de son auteur ${ }^{7}$. L'organisation macrostructurale de la matière est le résultat, dans certains cas, d'une sélection, mais surtout d'une classification des maladies à une période et pour un auteur donné, comme le rappelle Danielle Jacquart ${ }^{8}$. Le Lilium se compose de sept livres, dont chacun correspond à une région du corps ou à un type d'affection. Ils sont divisés en chapitres, eux-mêmes parfois subdivisés en sous-chapitres, dont le contenu se construit de la façon suivante : une définition, un exposé des causes puis des signes, la pose du diagnostic, les cures, et, enfin, une déclaration et/ou une clarification, c'est-à-dire une exposition ou discussion des doutes levés sur une question en particulier ou dus à une incohérence au sein du discours des autorités ${ }^{9}$. Ces usages sont autant d'aides à la lecture dans un contenu dense, ainsi que l'expression de la visée didactique de l'œuvre, qui donne les clés de son développement et facilite ainsi l'appréhension du discours médical. Ces intentions sont conservées par les copistes/traducteurs des fragments, mais leurs réalisations diffèrent ${ }^{10}$. Les deux imprimés sur lesquels nous élaborons notre réflexion recourent à des pratiques typographiques qui viennent souligner cette structuration de la matière. Le manuscrit A multiplie les aides à la lecture au sein des marges, et le manuscrit $\mathrm{B}$ a recours à la rubrication. Les fragments de $\mathrm{A}$ et $\mathrm{C}$ ne

\footnotetext{
7 Geneviève Dumas, Santé et société à Montpellier à la fin du Moyen Âge, Leiden/Boston, Brill, 2015, p. 180.

${ }^{8}$ Danielle Jacquart, «La pathocénose historique face à l'écran du cadre nosologique : l'exemple du Moyen Âge occidental», dans Le Concept de pathocénose de M. D. Grmek. Une conceptualisation novatrice de l'histoire des maladies, dir. Joël Coste, Bernardino Fantini et Louise Lambrichs, Paris, Droz, 2016, p. 203-229.

${ }^{9}$ Définition établie à partir de l'entrée Clarification du DMF : Dictionnaire de Moyen français [(DMF 2015) http://www.atilf.fr/dmf, ATILF - CNRS \& Université de Lorraine] et du Dictionnaire du français Scientifique Médiéval (DFSM) issu du projet CREALSCIENCE-STIH/Paris-Sorbonne.

${ }^{10}$ Aucun indice ne permet de distinguer de façon absolue le travail de copie du travail de traduction. Nous emploierons donc cette formule «copiste/traducteur» pour marquer cette indétermination.
} 
conservent que des portions restreintes - mais qui font unité- de l'ensemble du Lilium, là où le manuscrit $\mathrm{B}$ déstructure et réagence les fragments du Lilium choisis. Le discours médical reste le même, mais on voit se dessiner des intentions divergentes, certainement en vue d'emplois différents ou d'emplois similaires, mais par des lectorats différents.

Le phénomène d'énumération se rencontre à plusieurs niveaux du fait de cette volonté de totalisation du savoir médical de l'auteur. Nous distinguons les listes juxtaposant un ensemble d'objets dans le but de passer en revue un nombre fini ou non de possibles et les structures de déploiement, qui partent de cette accumulation d'objets pour développer chacun d'eux.

\section{Des indices sur les enjeux de traduction et de composition}

Pour ce premier cas, nous relevons les listes de patients types et/ou de lecteurs potentiels, mais aussi des listes de villes, d'animaux, de maux, d'ouvrages complémentaires, d'auteurs et enfin de préparations médicinales. En voici quelques-unes :

Item l'air doibt estre cler et net. Et se on n'a tel air naturellement, on le doit faire artificiellement, mesmement es cités ou est air obscur chault et moiste qui sont assises sur grans fleuves, si comme Rome dame des provinces, Lyon, Arles, Paris, et Coulogne et ainsi des aultres ${ }^{11}$.

Morsure venimeuse c'est solution de continuité faite de aucune beste venimeuse, si comme sont serpens, araignés, escorpions et vespes, aultres bestes venimeuses semblables qui habitent et demourent prés de nous ${ }^{12}$.

\footnotetext{
${ }^{11}$ Imprimé de 1495 - Livre 5, chapitre 8, «C'est le traité de regime de santé », sousensemble du chapitre « De fastide », p. 336a.

${ }^{12}$ Imprimé de 1495 - Livre 1, chapitre 14, « le XIIII c'est de morsure de serpent et des aultres bestes venimeuses », p. 42a-42b.
} 
Le second parle des passions et maladies de la conjunctive et sont .XIII. c'est assavoir obtalmie, ongle, macule, rougete, pannicule, pouldre qui chiet es yeulx, duresse, prurite, apostume, graveleux ulcere, et sanie, excressence de chair et diminucion, et fistulle ou lacrime ${ }^{13}$.

Item fievres ont quatre temps, si comme ont les aultres maladies, c'est assavoir commencement, accroissement, estat et declinacion ${ }^{14}$.

Parmi ces quatre exemples, les deux derniers sont des listes achevées ou fermées et s'opposent aux deux premiers, listes inachevées ou ouvertes, qui rappellent ce goût particulier pour l'effet de liste au Moyen Âge. Ces listes, d'une part, précisent l'énoncé par l'insertion d'un système analogique et, d'autre part, posent le cadre du développement par la dénomination de l'ensemble des référents nécessaires. Le dernier exemple met en évidence deux marques du développement syntaxique des listes selon les deux enjeux définis : «si comme» et «c'est assavoir ». La locution conjonctive «si comme » introduit un système comparatif d'égalité entre le référent du contexte antécédent, ici les fièvres, et le référent externe qu'il introduit, les autres maladies. Comme le montre Annie Kuyumcuyan, le système comparatif établi permet de convoquer des réalités extérieures au discours et enrichit l'énoncé en se fondant sur «la mémoire discursive, c'est-à-dire l'ensemble des connaissances jugées nécessaires par le locuteur/scripteur pour que l'interlocuteur interprète de manière adéquate son énoncé ${ }^{15} »$. Ces listes inachevées relèvent d'une manifestation de la volonté d'exhaustivité, volonté de totalisation du savoir qui avoue ses limites par des

\footnotetext{
${ }^{13}$ Imprimé de 1495 - Table du livre 3, Cy commence le tiers livre qui parle de la maladie des yeulx... [Titre du chapitre 2], p. 184b.

${ }^{14}$ Imprimé de 1495 - Livre 1, chapitre 1, «Le premier chapitre parle de fievre en general », p. 2 b.

${ }_{15}$ Annie Kuyumcuyan, «Comme et ses valeurs : le point de vue historique (XIV ${ }^{\mathrm{e}}$ $\mathrm{XVI}^{\mathrm{e}}$ siècles) », Langue française, 149, 2006, p. 125.
} 
interruptions au moyen de ces tours résomptifs, comme «et ainsi des aultres ». Le recours à des tours résomptifs - qui viennent arrêter l'énumération - montre l'incapacité du discours à prendre en charge la totalité du système analogique, mais il met surtout en évidence le travail de sélection des realia convoqués, qui s'accompagne d'un travail de définition par l'exemple. Ainsi, dans la seconde citation, la liste précise le référent sur lequel s'élabore le système analogique, et sa conclusion achève de définir ce référent par l'évocation du milieu de vie du lecteur : «aultres bestes venimeuses semblables qui habitent et demeurent prés de nous ». La locution «c'est assavoir » sert à introduire les dénominations des référents d'un nouveau développement. Elle a une fonction métalinguistique : elle décrit et explicite le discours à venir, en établissant une mise en relation de l'ensemble introduit, de façon univoque, au référent sur lequel il se construit. Cependant nous ne sommes pas là dans la simple équivalence, mais dans la nécessité d'une augmentation de l'énoncé initial par sa description. Cette description, ici à caractère didactique, s'opère par la mobilisation d'un lexique scientifique afin de déterminer les étapes de la fièvre.

Les fragments manuscrits peuvent conserver ces énumérations, malgré l'enregistrement de variantes. Tout d'abord, les énumérations dont les variantes ne conduisent pas à des interprétations différentes :

[A] Donc le medicin qui sera diligent, pourra faire dez choses devant dittez ou d'aucunes d'icellez sirop, electuaire, confiture, trocisque, pouldre, unguent, oli, emplaistre ou choses semblablez ${ }^{16}$.

[B] Pourquoy doncques le mege, qui sera saige et diligent, pourra avec ces materiaux, de ung ou de pluseurs, faire syrop ou electuaire ou

\footnotetext{
${ }^{16}$ A (BnF, fr. 1288) - Livre 7, chapitre 21, Antidotaire, du Lilium intégral en français, sous ensemble « Medicine contre fievre fleumatique », f. 139va-139vb.
} 
trocisques ou pouldre ou unguemens ou emplaistres et leurs semblables a ce appries ${ }^{17}$.

[D] Et le medecin poura faire de ces choses ou de une ou de plusieurs, ou sirop ou electuaire que on dit troncz, pouldres, unguens, emplastres, huilles et les semblables ${ }^{18}$.

Cette accumulation de formes médicamenteuses, dont l'amplitude est sans conséquence, relève peut-être d'un goût manifeste pour la liste, comme nous venons de le mentionner, mais qui semble apparaître ou au contraire être absent de ces témoins. Ainsi, le manuscrit A semble plus concis que le manuscrit $\mathrm{B}$, ce que confirme l'exemple suivant :

[A] Item veci une medicine qui vault aux medicins pour cognoistre lez urinez et pour barbiers pour bien frappez ou milieu de la vaine, et pour bien lire lettre delié ${ }^{19}$.

[B] Medecine qui est ordonnee et vault aux medecins, a ce qu'ilz puissent veoir le contenu de toutes medecines et urines et qu'ilz saichent bien discerner et declarer des coulleurs. Et si vault aux barbiés, ad ce qu'ilz puissent frapper leur lance au milieu de la vene. Et si vault aux lisans, aux religieux et autres gens, a ce qu'ilz saichent lire toutes meimes heures et minutes, et aussi vault aux escripvains ${ }^{20}$.

[D] Vecy medecine qui vault aux medecins pour mieulx veoir ce qui est contenu dedens l'orine et affin que ilz puissent mieulx juger des couleurs. Et se vault aussi aux barbiers et cirurgiens pour mieulx ferir en la veine au millieu. Et vault aux

\footnotetext{
${ }^{17}$ B (BnF, fr. 1327) - Livre 7, chapitre 21, Antidotaire, du Lilium intégral en français, «Pillulles ordonnees pour evacuer eaue citrine de ydropisie et des ydropiques qui sont ainsi faictes », f. 12 r.

${ }^{18}$ Imprimé de 1495 - Livre 7, chapitre 21, Antidotaire, sous ensemble «Contre ydropizie », p. 487a.

${ }^{19}$ A (BnF, fr. 1288) - Veci ung petit tractié qui parle dez passions depuis le chief jusquez aux piez maistre Bernard de Gourdon, Livre 7 chapitre 21, Antidotaire, du Lilium intégral en français sous ensemble « De doulour dez yeulx », f. 137va.

${ }^{20}$ B (BnF, fr. 1327) - Livre 7, chapitre 21, Antidotaire, du Lilium intégral en français, «Le premier chappitre est de la maladie, douleur et passion des yeulx en tout ou en partie $»$, f. $4 \mathrm{v}$.
} 
maistres lecteurs qui lysent et aux moynes et aux aultres qui lisent lettres menues ${ }^{21}$.

Cette introduction à la cure mentionne les différentes professions pouvant avoir besoin du remède. On retrouve le pragmatisme inhérent au manuscrit A, copie synthétique, et l'amplification propre au manuscrit B, là où la tradition imprimée fait office d'intermédiaire. Notons que l'imprimé fait mention des « maistres lecteurs », soit des universitaires, là où $\mathrm{B}$ préfère les «escripvains ». Le manuscrit $\mathrm{A}$ ne retient que les professionnels de santé : «medicins » et «barbiers ». On voit apparaître dans ces variantes des indices sur les copistes/traducteurs et/ou le lectorat envisagé. Les choix de traduction sont donc des indices sur la personnalité du traducteur et des lecteurs potentiels, par exemple dans la comparaison des deux citations suivantes sur les temps d'évolution des fièvres :

[B] Aussi la fievre se fait ensemble les autres maladies selon les quatre temps. Et aussi sont des maladies ainsi comme est le commancement, la augmentacion ou acroissement, l'estat et la declinacion, selon les differances des constitucions generalles et specialles. Il fault scavoir l'estat de la fievre et la congnoissance du temps des maladies, ainsi comme il est distingé de la part digestive et proclamé ; et qui le veult bien scavoir voise a nostre livre de pronostiquer, auquel lieu, au plaisir de Dieu, vous pourrez veoir et congnoistre les differances, ainsi comme dient Galien, Avicene et pluseurs autres ${ }^{22}$.

[D] Item fievres ont quatre temps, si comme ont les aultres maladies, c'est assavoir commencement, accroissement, estat et declinacion. Et qui vouldra scavoir les

${ }^{21}$ Imprimé de 1495 - Livre 7, chapitre 21, [Antidotaire], sous ensemble «Des maladies des yeulx », p. 482b et 483a.

22 B (BnF, fr. 1327) - Livre 1, chapitre 1 du Lilium intégral en français, "Le $\mathrm{XVI}^{\mathrm{e}}$ chappitre est de fievre en general », f. 12r. 
differences et les gendres et les manieres et aussi toutes les especes de fievres et la congnoissance des temps des maladies ainsi que elles sont distinguees et divisees par la digestion et par les peroximes, si voyse a nostre livre de $\mathrm{p}$ [ro]nosticacion, car en ce livre la, par la grace de Dieu, nous avons mis et assemblé les ditz de Avicene, de Galien et de Ypocras et des aultres, dont nous voulons commencer a fievre effimere $^{23}$.

La variation dans l'énumération des autorités, qui relève de l'effet de sélection inhérent à la transmission du texte, est un phénomène commun des textes de savoir. Dans l'énumération des temps de fièvre, par contre, on voit un effort de traduction différent entre la version de l'imprimé et celle du manuscrit. Une première différence mineure est à noter, l'interversion entre la mention de l'objet de l'énumération, soit les quatre temps, et la comparaison, soit les «aultres maladies». Plus intéressant, le latin «Item», le plus généralement conservé comme articulation du discours et aide à la lecture dans les traductions françaises, est traduit par le français «aussi» dans le manuscrit B. Enfin, on remarque des choix de clarification du discours au niveau lexical par l'apparition d'un doublet synonymique dans la version manuscrite ou par le recours à une nouvelle énumération dans le cas de l'imprimé énumération tronquée dans le manuscrit $\mathrm{B}$. Ces variantes sont des enrichissements, elles résultent des interprétations du texte latin par le copiste/traducteur et d'une réflexion sur la lisibilité, sur la compréhension $\mathrm{du}$ texte par le lectorat. Enfin, si nos fragments n'ont pas d'amples prologues de traducteur, les listes sont des espaces pleinement exploités qui justifient la réorganisation du Lilium en fragment :

\footnotetext{
${ }^{23}$ Imprimé de 1495 - Livre 1, chapitre 1, «Le premier chapitre parle de fievre en general », p. 2b-3a
} 
[A] Item tu dois entendre que par la grace de Dieu, nous avons ordonné pour cause de ceste heuvre cy escripte, aucuns tractiez et qui lez met tout ensemble, ce seroit euvre parfaicte : le livre dez regimes des maladies agues, item le livre de crisis et dez jours cretiques, item le livre qui sera entitulé tabula ingeniorum, car sans luy ne peut l'en riens faire, item le livre de graduacion $^{24}$.

[B] Et devez nocter et entendre que nous avons ordonné par la grace de Dieu quatre ordonnances des complections des quatre temps, qui ont esté par moy longue espace de temps experimenteez avant ce que j'aye voulu encommencer ceste euvre, lesquelles se elles estoient icy adjoustees l'euvre seroit parfaicte. Dont la premiere est la maistrise et science de l'aguille, de la maladie et du libeau de crisis et des jours critiques, et du libeau qui se intitule a la table des engins et du gouvernement de cyreurgie, et les libeaux de la graduacion de chascun grant selon la disposicion du concurrant, ainsi comme ung chascun le doit savoir et congnoistre. Tous lesquelz libeaux sont neccessaire assavoir a chascun cyreurgient qui veult pratiquer en tout ar de surgie. Pourquoy ung chascun preingne garde audit, car selon ce qu'il vouldra user audit art pour avoir et acquerir honneur et proffit en la science de sireurgie selon ce qu'il est dessus desclaré ${ }^{25}$.

Dans cet exemple, nous notons une belle amplification de l'énumération des œuvres citées, avec une mention particulière, celle des «quatre ordonnances des complections des quatre temps». Cette mention, qui n'apparaît pas dans le manuscrit A, est une invention du manuscrit B qui insère au milieu des fragments choisis du Lilium

\footnotetext{
${ }^{24}$ A (BnF, fr. 1288) - Livre 7 chapitre 21, Antidotaire, du Lilium intégral en français, «Pour faire belle face et bel cuir », f. 140va-b.

${ }^{25}$ B (BnF, fr. 1327) - Livre 7 chapitre 21, Antidotaire, du Lilium intégral en français, «Medecine ordonnee pour donné couleur et rougeur aux pommes des jouez qui est telle », f. 15 r.
} 
medicinae, quatre chapitres du Liber de pronosticis de Bernard de Gordon - unique traduction française par ailleurs de cette œuvre qui nous soit parvenue. On retrouve la concision propre au manuscrit A, qui semble se placer dans une approche plus pratique de la médecine que le manuscrit B, beaucoup plus expansif.

\section{L'énumération comme support de développement}

Le second effet de liste part d'une énumération des objets pour déployer la liste et expliciter son contenu. Ce procédé d'accumulation terminologique puis de déploiement explicatif est à l'image des titres de chapitres qui donnent la liste des pathologies traitées en amont du développement. Le titre du chapitre 2 du livre 3 cité plus haut en est un bon exemple ${ }^{26}$. Ce procédé se rencontre aussi le plus souvent dans l'exposé des causes et des signes, soit la première moitié de chaque unité de contenu (chapitres ou sous-chapitres selon la partition en cinq ou six temps de développement). Ces déploiements peuvent faire l'objet de nouvelles accumulations ou de véritables explicitations. Nous appuierons notre analyse sur la «Douleur du chief » du livre 2, chapitre 10 et le cas des fièvres dite effimera du livre 1, chapitre 2.

Dans le livre 2, chapitre 10, «Douleur du chief », l'exposé des causes fonctionne sur ce principe en deux temps : le premier résume le propos sous la forme d'une énumération; le second est un déploiement de cette liste, pour expliciter chaque entité mentionnée. On passe ainsi de catégories génériques à un ensemble de sous-catégories, où sont

\footnotetext{
26 «Le second parle des passions et maladies de la conjunctive et sont .XIII. c'est assavoir obtalmie, ongle, macule, rougete, pannicule, pouldre qui chiet es yeulx, duresse, prurite, apostome graveleux, ulcere, et sanie, excressence de chair et diminucion, et fistulle ou lacrime. » Imprimé de 1495- Table du livre 3, Cy commence le tiers livre qui parle de la maladie des yeulx, [Titre du chapitre 2], p. $189 \mathrm{~b}$.
} 
rappelées les définitions principales ou des détails permettant de préciser les définitions et d'expliciter le contenu :

Douleur de chief, c'est accident de male complexion. Cause. Les causes de douleur du chief sont trois: male complexion diverse, solucion de continuité en l'un et l'autre. Les divisions particulieres des causes de douleurs du chief sont .XII. : aulcunes sont de choses fouraines, aulcunes de dedens, aulcu[n]es sont avec matiere, aulcunes sans matiere, aulcune douleur est maladie par elle, aulcune par accident, aulcune douleur vient de cause privee du cervel et aulcunes vienent de cause lontaine, aulcune douleur vient de dedens, aulcune de dehors, aulcune douleur contient tout et aulcune partie, aulcune est forte, aulcune foible, aulcune vient soudainement ou temps de santé, aulcunes non, aulcunes ensuyvent aulcunes maladies, aulcunes non, aulcunes est nouvelle, aulcune vielle, aulcune avec reume, aulcune non. Donc douleur aulcune est des causes extrinseques et sont plusieurs telles causes et sont ramenees a .XIII. La premiere, c'est cheoir, ferir ou hurter et les semblables. La seconde peut estre chaleur de souleil ou de feu ou d'air en temps d'aust ou unguent. La tierce peut estre grant froidure de dehors ou d'air ou d'eaue ou de unguens et les semblables. La quarte cause est secheur d'air ou de unguens etc. La quinte, c'est trop grant humidité d'eaue ou de pl[ui]es ou de unguens. La .VI., c'est du vent qui souffle fort et mesmement de midi et de septentrion. La .VII., ce sont vapeurs fouraines eslevees des choses pudentes, si comme charongne et assa fetida, ou de choses aromatiques comme fleurs de lis, rozes ou musquet, ou quant on regarde les cavernes et minieres parfondes, et les semblables. La .VIII., c'est luy trop esmouvoir, comme ire couroux ou fort courrement, trop labourer ou rafaictier trop. La .IX. cause peut estre trop fort dormir et deshordonnés, ou trop veiller. La .X., c'est baignier superfluement 
mesmement en eaue sulphuree. La .XI., c'est inanicion ou replecion trop grande ou crapule ou yvrongnie ou menger viandes fumeuses, si comme aux cibes et fort vin. La douziesme peult estre son ou cris terribles, comme tonoire etc. La treziesme peult estre trop fort couvrir le chief se on le continue ${ }^{27}$.

Le discours fait l'objet d'une structuration rigoureuse, il opère comme un véritable système basé sur l'énumération. Dans la première liste des causes, nous notons ainsi la prédominance de la forme «aulcun» dont la répétition souligne la juxtaposition des éléments composant la liste. La numération permet de marquer le passage entre chaque type de cause. Enfin, la présence des conjonctions de coordination « et » et «ou », qui soutiennent la spécification du système référentiel, rythme le discours. La première liste a un but descriptif, la seconde aura une visée plus large: descriptive, explicative et même parfois prescriptive. Les listes fermées font la synthèse de la matière à déployer, et peuvent mener, dans ces déploiements au recours à la liste ouverte le plus généralement inachevée.

Le chapitre 2 du livre 1 sur les «fievre effimere » présente une structure de développement sur les causes beaucoup plus restreintes. Ce sont les signes qui nous intéresseront ici :

Les signes de fievre effimere, aucuns sont generaulx aucuns sont especiaulx. Vecy les generaulx. Effimere ne vient pas par choses antecedentes, mais par choses primitives et communement elle ne vient pas avec rigour. Le poulx et l'orine ne sont pas trop bons de chaleur naturelle, ne la chaleur n'est pas pongitive ne mordicative au taster ains est ainsi que chaleur de homme couroucé ou qui a trop labouré, et communement elle se termine de sueur humide naturelle. Et dit Avicene que se on met ung tel

${ }^{27}$ Imprimé de 1495 - Livre 2, chapitre 10, « De douleur du chief », p. 121a et b. 
malade en ung baing et qu'il ne luy viengne aucune opilation ou hirsure, c'est signe de effimere. Vecy les signes especiaulx. Se effimere vient par chaleur de soleil ou par chose semblable, adonc la teste est chaulde et les yeulx. Et se on taste la face le cuir et le poulz, ilz se debatent et esmeuvent de plus en plus. Mais se la fievre vient par trop grant froidure, la beaulté de la face se mue et devient livide. Et s'elle est causee par choses stipiques et opilatives, on sent le cuir sec et ainsi comme estendu. Et s'on sent le cuir, on sent ainsi que chaleur occulte. Et se on tient longuement la main sur le cuir, on sent chaleur ague. Et se elle vient par viandes ou par beuvrages aguz, on sent chaleur entour la region du foye et l'orine est tainté. Et s'elle vient par labeur et par travail, on a foiblesse par tout le corps et douleur es joinctures. Et se c'est par ire ou par couroux, les yeulx sont apparans et engrossis et la face est rubiconde. Et se c'est par tristesse, les yeulx sont concavez et enfossez. Et se c'est par luxure, on sent les choses de dedens chaudes estendues et desicatives. Et se ces signes si especiaulx ne souffisent, nous recourrons au jugement du pacient ${ }^{28}$.

L'énumération des signes relève d'un déploiement similaire à ce qu'on observe pour le développement des causes, avec une mise en évidence des temps de progression du discours. On a ainsi une première division en «signes generaux » et «signes especiaulx ». Ces deux temps sont introduits par le présentatif «vecy». On relève enfin dans le développement de ces types de signes, un système de répétition marquant la progression, soit le coordonnant suivi de l'adverbe « communement », puis le coordonnant « et » suivi de «si » ou de «se ». De ce chapitre 2, le manuscrit B n'a retenu que la partie dite des cures, dans une version

\footnotetext{
${ }^{28}$ Imprimé de 1495 : Livre 1 chapitre 2, «Cy parle de une fievre qui s'appelle effimere » p. 3a-4a.
} 
abrégée qui plus est. Le chapitre s'appelle alors « le XVII ${ }^{e}$ chappitre est de fievre dicte effimera urage » suivant la numérotation des chapitres dans la réorganisation par le copiste/traducteur. Ce chapitre 17 reprend malgré tout en tête la définition de la « fievre effimere » et l'annonce de la structure du chapitre telle qu'on peut la rencontrer dans les imprimés.

Commençons par nous intéresser au fond. Les causes nécessaires à l'appréhension de ces cures sont réduites à trois temps d'opération : on trouve ainsi la cause par excès de chaud, la cause dite froide, puis des cures en temps de «declinacion». Les considérations concernent alors l'alimentation, les bains, la crapule, la distinction entre fièvre dite putride et celle dite éphémère, comme dans les imprimés, mais on note l'absence des considérations sur l'air ou encore la «catarre ». On a ainsi une restriction de la matière à des fins pratiques immédiates, c'est-à-dire une réorientation de l'intérêt du chapitre par un recentrement sur des points particuliers.

En ce qui concerne la progression du discours au sein de cet ensemble de cure, on constate des similitudes avec les imprimés, notamment par la prédominance de la coordination, avec le coordonnant « et », des marqueurs d'opposition syntaxique «mais » et «car» ou l'adverbe explicatif «pourquoy» et enfin le recours aux présentatifs, dont la forme «c'est», modalisé ou non par les formes relatives à la conjonction «si », tel que : «ce c'est». Comme pour le cas des deux conjonctions de coordination « et » et «ou » mentionnées plus haut, ces marqueurs de progression du discours sont maintenus malgré le passage à l'imprimé et l'abondante ponctuation qui se déploie dans notre texte par ce medium de transmission. La langue des manuscrits est presque exempte de ponctuation. Celle-ci, lorsqu'elle est présente, suit un mouvement de pensée du copiste/traducteur, ou bien souligne une articulation du discours en particulier, en dehors des ressorts syntaxiques. 
L'abondante présence des coordonnants et d'autres marqueurs de progression du discours, tels que les présentatifs, sont des jalons de structuration du développement et en guident l'appréhension. Ce balisage de la langue par la répétition de ces outils syntaxiques fait apparaître le squelette de la progression et structuration du discours en tant que ressort d'une rhétorique didactique. Notons que ce balisage n'est pas abandonné dans les versions imprimées, malgré la présence plus massive de la ponctuation qui oscille entre stratégie syntaxique et soulignement de l'organisation des idées. La langue à l'œuvre pourrait ainsi être perçue comme un véritable art d'opérer l'ordonnancement de la matière au niveau syntaxique.

Liste d'objets achevée ou inachevée, avec déploiement ou non, ce procédé est un enjeu principal d'accès au savoir et de sa structuration dans le Lilium medicinae. Manifestation de la volonté didactique et d'exhaustivité de la part de l'auteur, l'énumération est aussi un lieu dont la variation permet d'appréhender les enjeux de la traduction, soit les procédés et les intentions du copiste/traducteur, mais aussi emplois supposés des supports et/ou du lectorat. Ce procédé est un outil de structure textuelle, en tension entre l'efficacité et la concision que permettent la liste fermée - et son déploiement potentiel - et l'infinité des possibles supposés par les listes inachevées. La liste a ainsi une fonction rhétorique au sein du discours, mais elle est aussi un mode d'organisation de ce même discours et de la matière médicale. C'est un véritable système d'exposition qui se déploie, permettant la création d'une arborescence, véritable modélisation de la matière qui permet ainsi son appréhension par le lectorat. Enfin, cette perspective sous-tend une méthode d'approche de l'analyse médicale : de la recherche des causes en 
relation avec l'observation des signes à un ensemble de diagnostics aboutissant au choix d'une cure. 